\title{
Chloroplast Genome Analysis of Two Medicinal Coelogyne spp. (Orchidaceae) Shed Light on the Genetic Information, Comparative Genomics, and Species Identification
}

\author{
Kai Jiang ${ }^{1,2,3} \mathbb{D}_{\text {, Li-Yuan Miao }}{ }^{1,4}$, Zheng-Wei Wang ${ }^{1}$, Zi-Yi Ni ${ }^{1}$, Chao Hu ${ }^{1,2}$, Xin-Hua Zeng ${ }^{1,2}$ \\ and Wei-Chang Huang 1,2,4,5,* \\ 1 Shanghai Chenshan Plant Science Research Center, Chinese Academy of Sciences, Chenshan Botanical \\ Garden, Shanghai 201602, China; jiangkai@csnbgsh.cn (K.J.); melodying_8829@163.com (L.-Y.M.); \\ wangzhengwei@csnbgsh.cn (Z.-W.W.); niziyi@csnbgsh.cn (Z.-Y.N.); huchao@csnbgsh.cn (C.H.); \\ zengxinhua@csnbgsh.cn (X.-H.Z.) \\ 2 Shanghai Key Laboratory of Plant Functional Genomics and Resources, Shanghai Chenshan Botanical \\ Garden, Shanghai 201602, China \\ 3 School of Ecological and Environmental Sciences, Shanghai Key Lab of Urban Ecological Processes and \\ Eco-Restoration, East China Normal University, Shanghai 200241, China \\ 4 College of Life, Shanghai Normal University, Shanghai 200234, China \\ 5 College of Landscape Architecture, Fujian Agriculture and Forestry University, Fuzhou 350002, China \\ * Correspondence: huangweichang@csnbgsh.cn
}

Received: 8 September 2020; Accepted: 5 October 2020; Published: 9 October 2020

\begin{abstract}
Although the medicinal properties of Coelogyne spp. have been previously studied, there is little genomic information providing a valuable tool for the plant taxonomy, conservation, and utilization of this genus. This study used the next-generation MiSeq sequencing platform to characterize the chloroplast (cp) genomes of Coelogyne fimbriata and Coelogyne ovalis. The Maximum Likelihood (ML) and Bayesian (BI) methods were employed to confirm the phylogenetic position of two Coelogyne species based on the whole chloroplast genome sequences. Additionally, we developed eight new primers based on the two $\mathrm{cp}$ genomes' medium variable regions and evaluated the transferability to another 16 Coelogyne species. We constructed phylogenetic trees including 18 Coelogyne species and four outgroup species using the chloroplast fragments with the ML method. Our results showed that the cp genomes of $C$. fimbriata and C. ovalis contained a small single-copy region $(18,839$ and $18,851 \mathrm{bp}$, respectively) and a large single-copy region $(87,606$ and $87,759 \mathrm{bp}$, respectively), separated by two same-length inverted-repeat regions $(26,675 \mathrm{bp}$ in C. fimbriata and $26,715 \mathrm{bp}$ C. ovalis, respectively). They all contained 86 protein-coding genes, 38 tRNA genes, and eight rRNA genes, revealing strong structure and gene content similarities. The phylogenetic analysis indicated a close relationship between the genera Coelogyne and Pleione. The newly developed primers revealed good transferability among the Coelogyne taxa and provided enough variable sites to distinguish C. fimbriata and C. ovalis. The two complete cp genomes and the eight new primers of Coelogyne provide new genomic data for further studies on phylogenomics, population genetics, and evolutionary history of Coelogyne taxa.
\end{abstract}

Keywords: Coelogyne; chloroplast genome; phylogeny; molecular identification

\section{Introduction}

Chloroplasts (cps) are photosynthetic organelles that play an essential role in providing energy for green plants [1]. The chloroplasts have their own genome. With a few exceptions, most chloroplast 
genomes consist of a single, large, circular DNA molecule, ranging in length from 120 to $160 \mathrm{~Kb}$, which contains two inverted repeats (IRs) that divide the molecule into a large single-copy section (LSC) and a small single-copy section (SSC) [2]. About 100-130 genes encode about 79 proteins, 30 transfer RNAs, and four ribosomal RNAs. The cp genomes show highly conserved gene content and order [3]. Furthermore, maternal inheritance is the primary mechanism for transferring chloroplastic genetic material between generations in most angiosperms [4]. No complicated recombination events occur in the chloroplast genome. Because of its haploid nature, its high conservation in terms of gene content and order, and its simple inheritance mode, the cp genome has been employed extensively in the study of phylogeography and in addressing evolutionary questions in plants.

Coelogyne Lindl. (Epidendroideae; Orchidaceae) is a genus comprising more than 200 species. It is widely distributed throughout Asia, including China, India, Indonesia, and the Fiji Islands. Its main centers of diversity are in the Himalayas, Sumatra, and Borneo [5]. Most species grow in tropical montane and lowland forest areas. Some species, which grow under cooler conditions, such as Coelogyne fimbriata and Coelogyne ovalis, prefer higher altitudes on mountains. These two species are epiphytic and grow on rocks or tree trunks, with slender and creeping rhizomes. They reproduce both sexually and by vegetative growth. One or two flowers can be found on a given scape. The flowers are nectarless and attract pollinators through fragrance. According to Cheng et al.'s report in 2009, C. fimbriata is food-deceptive and pollinated by worker wasps [6].

A few species in this genus have been identified as medicinal plants [7-9]. Especially in China, India, Nepal, and Thailand, people use Coelogyne species as traditional medicines. For example, an alcoholic extract of pseudobulbs from C. ovalis contained the phenanthrenoids, coelogin, and flavidin, with these substances showing spasmolytic activity [10]. Moreover, the whole plant of C. fimbriata is used to reduce "heat" (primarily, inflammation) [11]. However, there are many taxonomic issues to be addressed in the genus Coelogyne [12]. It is still debated as to whether the two species mentioned above should be merged into one species. To better understand the phylogeny and Coelogyne's species delimitations, we characterized the complete chloroplast genome sequences of $C$. fimbriata and C. ovalis. Using the two genomes, we developed eight primers for phylogenetic and delimited marker resources for future studies. Furthermore, we used these primers to amplify 18 Coelogyne species (including C. fimbriata and C. ovalis) to test the newly developed markers' efficacy and construct a robust phylogenetic tree to improve our understanding of Coelogyne species' relationship.

\section{Results}

\subsection{Genome Sequencing and Assembly}

Through the Illumina MiSeq sequencing, we obtained 3,041,719 and 3,624,370 clean reads from the Coelogyne fimbriata and Coelogyne ovalis's total chloroplast DNA. There were 2,804,465, and 3,374,288 reads the can map to the reference genome Calanthe sylvatica. The results indicated similar chloroplast content and structure between the Coelogyne and Calanthe chloroplast genome. The complete cp genome sequences of C. fimbriata (GenBank: MK946948) and C. ovalis (GenBank: MK946949) were 159,795 bp and 160,040 bp in length, respectively. Based on the C. sylvatica reference cp genome, the four junctions between LSC/IRs and SSC/IRs of the two Coelogyne species were validated by PCR-based Sanger sequencing, using four pairs of primers.

\subsection{The Organization of the Coelogyne Chloroplast Genome}

The chloroplast (cp) genomes of C. fimbriata and C. ovalis exhibited a typical quadripartite structure, consisting of a pair of inverted repeats (IRs) with similar length (26,7675 bp and 26,715 bp, respectively), separated by the Large single-copy $\operatorname{(LSC)}(87,606$ and $87,6759 \mathrm{bp}$, respectively) and Small single-copy (SSC) (18,839 and 18,851bp, respectively) regions. The whole cp genomes of the two species, showing the guanine-cytosine (GC) contents of the LSC, SSC, and IR regions, are shown in Figure 1. In C. fimbriata and C. ovalis, GC content was very similar at $37.4 \%$ and $37.3 \%$, respectively. However, the GC contents 
of the LSC and SSC regions in C. fimbriata ( $35.3 \%$ and $30.5 \%$, respectively) and C. ovalis ( $35.2 \%$, and $30.4 \%$, respectively) were markedly lower than those of the IR regions ( $43.3 \%$ for both species).
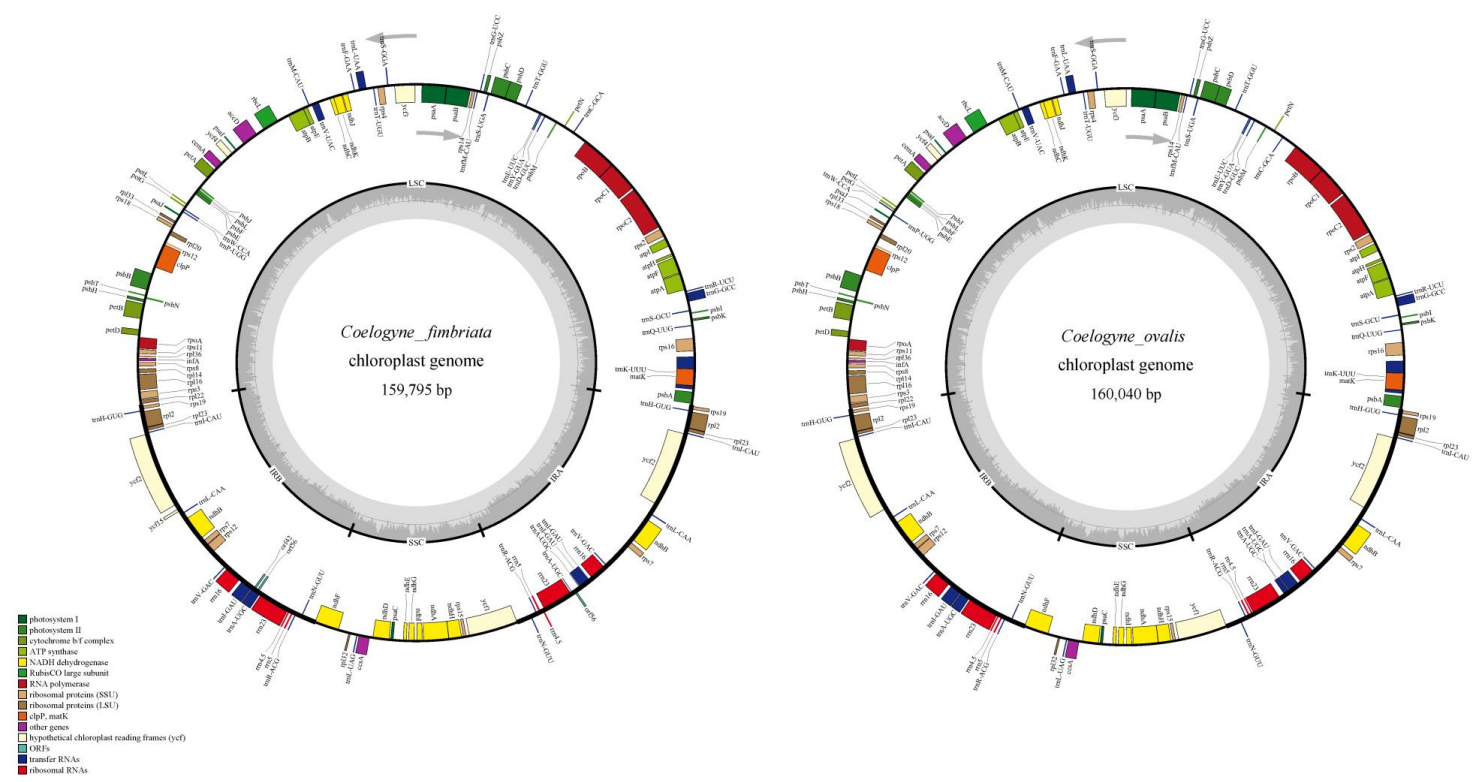

Figure 1. Physical maps of the complete chloroplast genomes in Coelogyne fimbriata and Coelogyne ovalis. The inner circle's genes are transcribed in the clockwise direction, while outside genes are counterclockwise. The areas with light and dark gray coloration in the internal circle suggest guanine-cytosine (GC) content of its genome.

Both cp genomes contained 86 protein-coding, 38 tRNA, and eight rRNA genes (Table 1). A total of 132 predicted functional genes were found through the annotation by DOGMA of the cp genome sequences of each of these two Coelogyne species. Of these, 115 genes were unique, including 81 protein-coding, 30 tRNA genes, and four rRNA genes (Figure 1, Table 2). The LSC region comprised 61 protein-coding genes and 21 tRNA genes, whereas 12 protein-coding genes and one tRNA gene were found in the SSC region. Eight protein-coding and eight tRNA genes were repeated in the IR regions. Among the 18 duplicated genes in the IR regions, six were protein-coding genes ( $n d h B, r p l 2, r p l 23$, rps7, rps19, and ycf2), eight encoded tRNAs (trnH-GUG, trnI-CAU, trnL-CAA, trnV-GAC, trnI-GAU, trnA-UGC, $t r n R-A C G$, and $t r n N-G U U)$ and four encoded rRNA (rrn16, rrn5, rrn4.5 and rrn23) (Table 1). Furthermore, the number of genes with introns was 16, including ten protein-coding genes and six tRNA-coding genes (Table 2). Among them, three of these genes contained two introns: the $\operatorname{clpP}, y c f 3$, rps12 genes, and a trans-spliced gene, rps12, with the $5^{\prime}$ end exon the LSC region and the intron $3^{\prime}$ end exon situated in the IR region (Table 3). 
Table 1. Characteristics and Basic Assembly Parameters of Two Coelogyne Chloroplast Genomes.

\begin{tabular}{ccc}
\hline Characteristics and Parameters & C. fimbriata & C. ovalis \\
\hline Raw reads (bp) & $3,142,569$ & $3,763,406$ \\
Clean reads (bp) & $3,041,719$ & $3,624,370$ \\
Average read length (bp) & 300 & 300 \\
Number of contigs & 1 & 1 \\
Total length of contigs (bp) & 159,795 & 160,040 \\
N50 length of contigs (bp) & 159,795 & 160,040 \\
Total cp genome size (bp) & 159,795 & 160,040 \\
LSC length (bp) & 87,606 & 87,759 \\
SSC length (bp) & 18,839 & 18,851 \\
IR length (bp) & 26,675 & 26,715 \\
Total CDS length (bp) & 79,891 & 78,258 \\
Total tRNA length (bp) & 2865 & 2911 \\
Total rRNA length (bp) & 9038 & 9041 \\
Total GC content (\%) & 37.39 & 37.35 \\
GC content for LSC (\%) & 35.30 & 35.20 \\
GC content for SSC (\%) & 30.50 & 30.40 \\
GC content for IR (\%) & 43.30 & 43.30 \\
Total number of genes & 136 & 133 \\
Protein-coding genes & 90 & 87 \\
rRNAs genes & 38 & 38 \\
tRNAs genes & 8 & 8 \\
Duplicated genes & 17 & 17 \\
\hline
\end{tabular}

Note, cp: Chloroplast; LSC: large single-copy region; SSC: small single-copy region; IR: inverted region; CDS: coding region; GC: guanine-cytosine.

Table 2. Gene Composition of the Coelogyne Chloroplast Genome.

\begin{tabular}{|c|c|c|}
\hline Categories of Genes & Groups of Genes & Name of Genes \\
\hline RNA genes & Ribosomal RNAs & 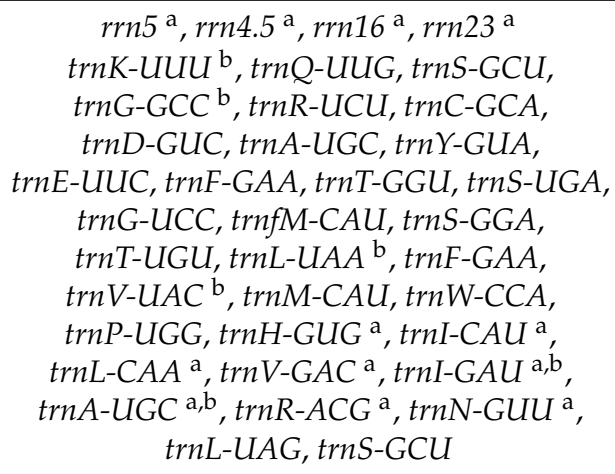 \\
\hline $\begin{array}{l}\text { Transcription- and } \\
\text { translation-related genes }\end{array}$ & $\begin{array}{l}\text { Small subunit of ribosome } \\
\text { Large subunit of ribosome } \\
\text { Transcription } \\
\text { Translation initiation factor }\end{array}$ & $\begin{array}{c}\text { rps2, rps3, rps4, rps7 } \mathrm{a}, r p s 8, r p s 11, r p s 12^{\mathrm{c}}, \\
r p s 14, r p s 15, r p s 16^{\mathrm{b}}, r p s 18, r p s 19^{\mathrm{a}} \\
r p l 2 \mathrm{a}, \mathrm{b}, r p l 14, r p l 16^{\mathrm{b}}, r p l 20, r p l 22, r p l 23^{\mathrm{a}}, \\
r p l 32, r p l 33, r p l 36 \\
\text { rpoA, rpoB, rpoC1 } \mathrm{b}, r p o C 2 \\
\operatorname{infA}\end{array}$ \\
\hline
\end{tabular}


Table 2. Cont.

\begin{tabular}{|c|c|c|c|c|c|c|}
\hline \multicolumn{2}{|c|}{ Categories of Genes } & \multicolumn{2}{|c|}{ Groups of Genes } & \multicolumn{3}{|c|}{ Name of Genes } \\
\hline \multicolumn{2}{|c|}{$\begin{array}{l}\text { Photosynthesis-related } \\
\text { genes }\end{array}$} & $\begin{array}{r}\text { NADH de } \\
\text { Phot } \\
\text { Photo } \\
\text { RubisCO } \\
\text { Cytochron } \\
\text { ATP } \\
\text { Cytochror }\end{array}$ & $\begin{array}{l}\text { rogenase } \\
\text { em I } \\
\text { subunit } \\
\text { complex } \\
\text { aase } \\
\text { synthesis }\end{array}$ & \multicolumn{3}{|c|}{$\begin{array}{c}n d h A^{\mathrm{b}}, n d h B^{\mathrm{a}, \mathrm{b}}, n d h C, n d h D, n d h E, n d h F, \\
n d h G, n d h H, n d h I, n d h J, n d h K \\
p s a A, p s a B, p s a C, p s a I, p s a J \\
p s b A, p s b B, p s b C, p s b D, p s b E, p s b F, p s b H, p s b I, \\
p s b K, p s b L, p s b J, p s b N, p s b T, p s b Z, p s b M \\
\operatorname{rbcL} \\
\text { pet } A, p e t B \mathrm{~b}, p e t D, p e t G, p e t L, p e t N \\
\operatorname{atp} A, \operatorname{atp} B, \operatorname{atpE}, \operatorname{atpF} \mathrm{b}, \text { atpH, atpI } \\
\operatorname{ccs} A\end{array}$} \\
\hline \multicolumn{2}{|c|}{ Others } & \multicolumn{2}{|c|}{$\begin{array}{l}\text { RNA processing } \\
\text { Carbon metabolism } \\
\text { Fatty acid synthesis } \\
\quad \text { Proteolysis }\end{array}$} & \multicolumn{3}{|c|}{$\begin{array}{l}\text { matK } \\
\operatorname{cem} A \\
\operatorname{accD} \\
\operatorname{clp} P^{\mathrm{c}}\end{array}$} \\
\hline \multicolumn{2}{|c|}{$\begin{array}{l}\text { Genes of unknown } \\
\text { function }\end{array}$} & \multicolumn{2}{|c|}{ Conserved reading frames } & \multicolumn{3}{|c|}{$y c f 1, y c f 2{ }^{\mathrm{a}}, y c f 4, y c f 3^{\mathrm{c}}, y c f 15, y c f 68^{\mathrm{d}}$} \\
\hline \multicolumn{7}{|c|}{$\begin{array}{l}{ }^{\mathrm{a}} \text { Gene with two copies; }{ }^{\mathrm{b}} \text { Gene with one intron; }{ }^{\mathrm{c}} \text { Gene with two introns. }{ }^{\mathrm{d}} \text { Gene existed in which species } \\
\text { chloroplast genome and copy number and intron number in each chloroplast (cp) genome. NADH: Nicotinamide } \\
\text { adenine dinucleotide. }\end{array}$} \\
\hline \multirow{2}{*}{ Gene } & \multirow{2}{*}{ Location } & \multicolumn{5}{|c|}{ Nucleotides in Base Pairs } \\
\hline & & Exon I & Intron I & Exon II & Intron II & Exon III \\
\hline $\operatorname{atpF}$ & LSC & $144 / 144$ & $965 / 964$ & $411 / 411$ & & \\
\hline $\operatorname{clp} P$ & LSC & $69 / 69$ & $963 / 950$ & $291 / 291$ & $675 / 673$ & $252 / 252$ \\
\hline$n d h A$ & SSC & $552 / 552$ & $1235 / 1235$ & $540 / 540$ & & \\
\hline$n d h B$ & IR & 777/777 & $701 / 710$ & $756 / 756$ & & \\
\hline petB & LSC & $6 / 6$ & $739 / 736$ & $642 / 642$ & & \\
\hline rpl16 & LSC & $9 / 9$ & $1007 / 1248$ & $399 / 399$ & & \\
\hline$r p l 2$ & IR & $387 / 387$ & $663 / 663$ & $432 / 432$ & & \\
\hline rpoC1 & LSC & $435 / 435$ & $766 / 778$ & $1617 / 1617$ & & \\
\hline $\operatorname{rps} 12^{\text {a }}$ & LSC & $126 / 126$ & - & $232 / 232$ & $549 / 549$ & $26 / 26$ \\
\hline rps16 & LSC & $40 / 40$ & $894 / 893$ & $248 / 248$ & & \\
\hline$y c f 3$ & LSC & $126 / 126$ & $721 / 723$ & $228 / 228$ & $672 / 672$ & $152 / 152$ \\
\hline $\operatorname{trn} G-G C C$ & LSC & $23 / 23$ & $700 / 700$ & $47 / 47$ & & \\
\hline $\operatorname{trnI-GAU}$ & IR & $42 / 42$ & $948 / 948$ & $35 / 35$ & & \\
\hline trnK-UUU & LSC & $37 / 37$ & $2915 / 2917$ & $26 / 26$ & & \\
\hline $\operatorname{trnL}-U A A$ & LSC & $35 / 35$ & $574 / 574$ & $50 / 50$ & & \\
\hline $\operatorname{trn} V-U A C$ & LSC & $39 / 39$ & $577 / 577$ & $35 / 35$ & & \\
\hline
\end{tabular}

a The $r p s 12$ is a trans-spliced gene with the $5^{\prime}$ end located in the LSC region and duplicated in the $3^{\prime}$ end in the IR regions. LSC: large single-copy region; SSC: small single-copy region; IR: inverted repeat region.

\subsection{Sequence Repeats}

The distribution, number, and type of microsatellites detected in the two cp genomes were analyzed. A total of 50 SSRs were found in C. fimbriata, of which 31 were in the LSC regions, whereas six and 13 were in the IR and SSC regions, respectively. On the other hand, in C. ovalis, there were 48 SSRs, with 34, four, and ten SSRs distributed in the LSC, IR, and SSC regions, respectively (Figure 2a). In addition, seven SSRs were discovered in the coding sequences (CDSs), 35 in intergenic spacers (IGSs), and eight in intron regions of the $C$. fimbriata $\mathrm{cp}$ genome, whereas the corresponding numbers in the C. ovalis cp genome were five in CDS, 32 in IGS and 11 in intron regions (Figure 2b). Among these SSRs in C. fimbriata and C. ovalis, mononucleotide repeats were the most frequent, accounting for $78 \%$ and $79 \%$, respectively, whereas dinucleotide repeats accounted for $20 \%$ and $19 \%$, respectively, with trinucleotide repeats accounting for $2 \%$ and $2 \%$, respectively (Figure $2 \mathrm{c}$ ). 


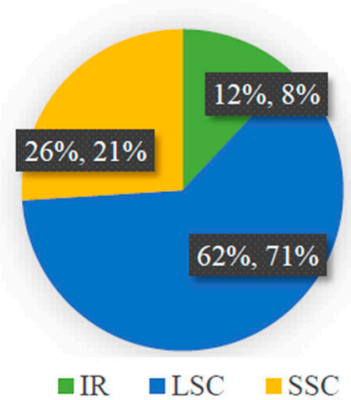

c

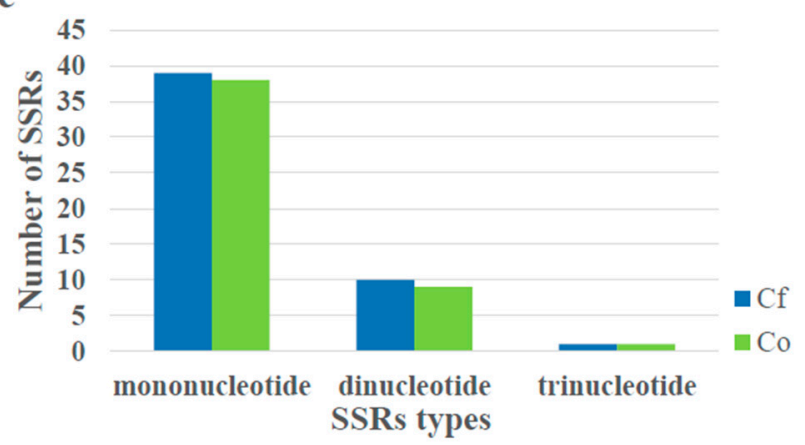

b

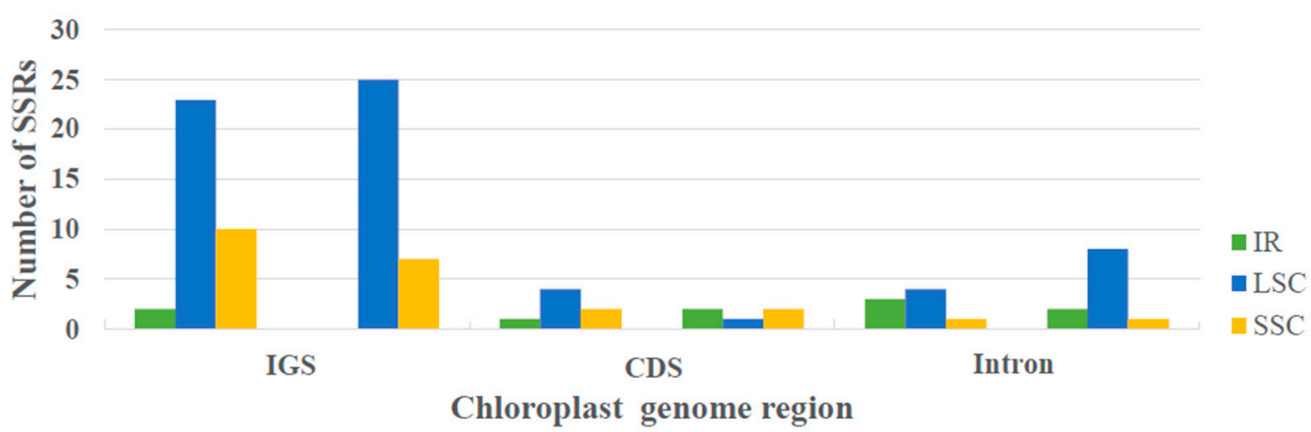

Figure 2. The distribution, type, and presence of simple sequence repeats (SSRs) in the chloroplast genomes of C. fimbriata (left) and C. ovalis (right). (a) Presence of SSRs in the regions of large single-copy region (LSC), small single-copy region (SSC) and inverted regions (IRs). (b) Presence of SSRs in the intergenic spacer (IGS), coding region (CDS), and Intron of LSC, SSC, and IRs regions. (c) Presence of the numbers of polymers.

Furthermore, 43 repeat sequences with different types and locations were identified in each of the two cp genomes. There were ten repeat sequences with motifs of one and ten with motifs of two in C. fimbriata, compared with 11 and six with motifs of one and two, respectively, in C. ovalis. The number of forward repeats was eight, and the number of palindrome repeats was 11, and there were no reverse or complementary repeats in C. fimbriata, whereas there were four, 11 , and two forward, palindrome and reverse repeats, respectively, in C. ovalis. Of these repeats, $65 \%$ were in the same regions of the two species, with the remainder of them existing in different regions in C. fimbriata and C. ovalis.

\subsection{Comparative Genome Analysis}

A total of 271 polymorphic sites can be found by comparing C. fimbriata and C. ovalis cp genomes. The nucleotide diversity $\left(P_{\mathrm{i}}\right)$ was 0.0017 between the above $\mathrm{cp}$ genomes. According to the comparison among the six Orchidaceae species representing Apostasioideae, Vanilloideae, Cypridoideae, Orchidoideae, and Epidendroideae, we found that Apostasioideae is very different from the other Orchidaceae species in genomic structure and gene contents. However, other species except Apostasia shenzhenica showed similar genomic structure and gene contents (Figure 3). We chose C. sylvatica to be the reference genome. The mVISTA tool was used to perform the comparative analysis of cp genome sequences in three species: C. fimbriata, C. ovalis, and C. sylvatica (Figure A1). From the results, we could see that the IRs showed higher sequence conservation between species than did the LSC and SSC regions. 


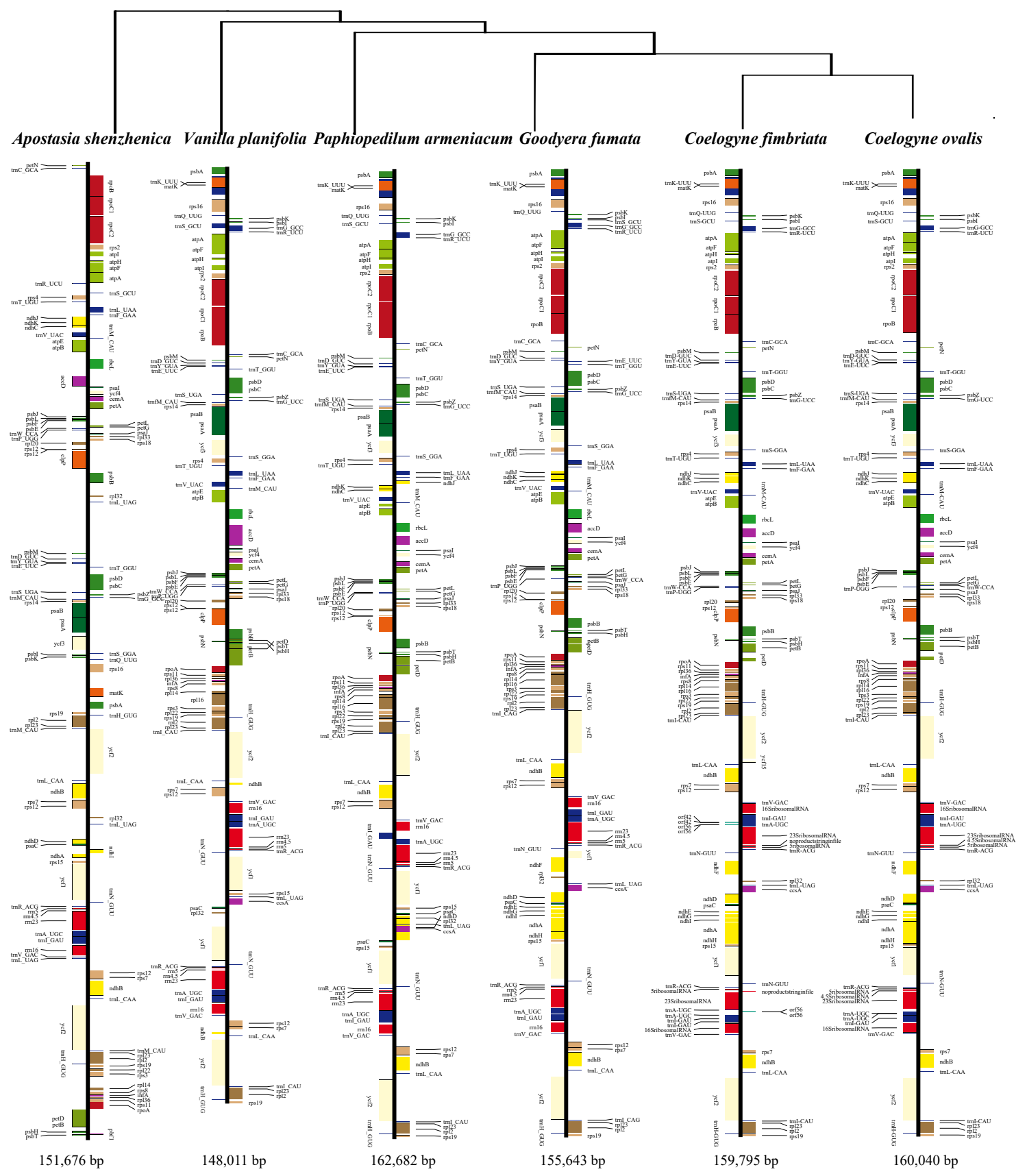

Figure 3. Comparative chloroplast genomes of six Orchidaceae species representing Apostasioideae (Apostasia shenzhenica), Vanilloideae (Vanilla planifolia), Cypridoideae (Paphiopedilum armeniacum), Orchidoideae (Goodyera fumata), and Epidendroideae (Coelogyne fimbriata and Coelogyne ovalis), respectively.

Furthermore, the non-coding regions were revealed to be less highly conserved than the coding regions, with most of the divergences being in the IGSs. The boundary regions of these three species were also compared (Figure 4). The rpl22 gene extended from the LSC to the inverted repeat region B (IRb) region by $76 \mathrm{bp}$ in C. sylvatica but by $37 \mathrm{bp}$ in both C. fimbriata and C. ovalis. At the boundary of IRb/SSC, the main part of the $n d h F$ gene in C. sylvatica was in the SSC region, with 60 bp located in the IRb region, compared with $68 \mathrm{bp}$ in each of the other two Coelogyne species. The ycf1 gene was $1031 \mathrm{bp}$ and $16 \mathrm{bp}$ from the borderline between SSC and the inverted repeat region A (IRa) in C. sylvatica and C. fimbriata, respectively, whereas it was present in the SSC region in C. ovalis, at 348 bp 
from the SSC/IRa borderline. The $r p s 19$ and $p s b A$ genes were distributed in the edge regions of the $\mathrm{IRa} / \mathrm{LSC}$ boundary line in all three species, with the distance from these two genes, $r p s 19$ and $p s b A$, to the boundary line between IRa and LSC being $259 \mathrm{bp}$ and $103 \mathrm{bp}$, respectively, in C. sylvatica, $128 \mathrm{bp}$ and $103 \mathrm{bp}$ in C. fimbriata, and $122 \mathrm{bp}$ and $109 \mathrm{bp}$ in C. ovalis. With C. sylvatica as the reference genome, we found that the rpl22 gene moved away from LSC/IRb boundary line to the LSC region, whereas the ycf1 gene shifted from the SSC/IRa boundary line to the SSC region, with genes like $n d h F$ and rps19 moving to the boundary line of IRb/SSC and IRa/LSC, respectively. Moreover, the $p s b A$ gene made a slight (6 bp) movement back to the LSC region in C. ovalis, compared with C. fimbriata and C. ovalis (Figure 4).

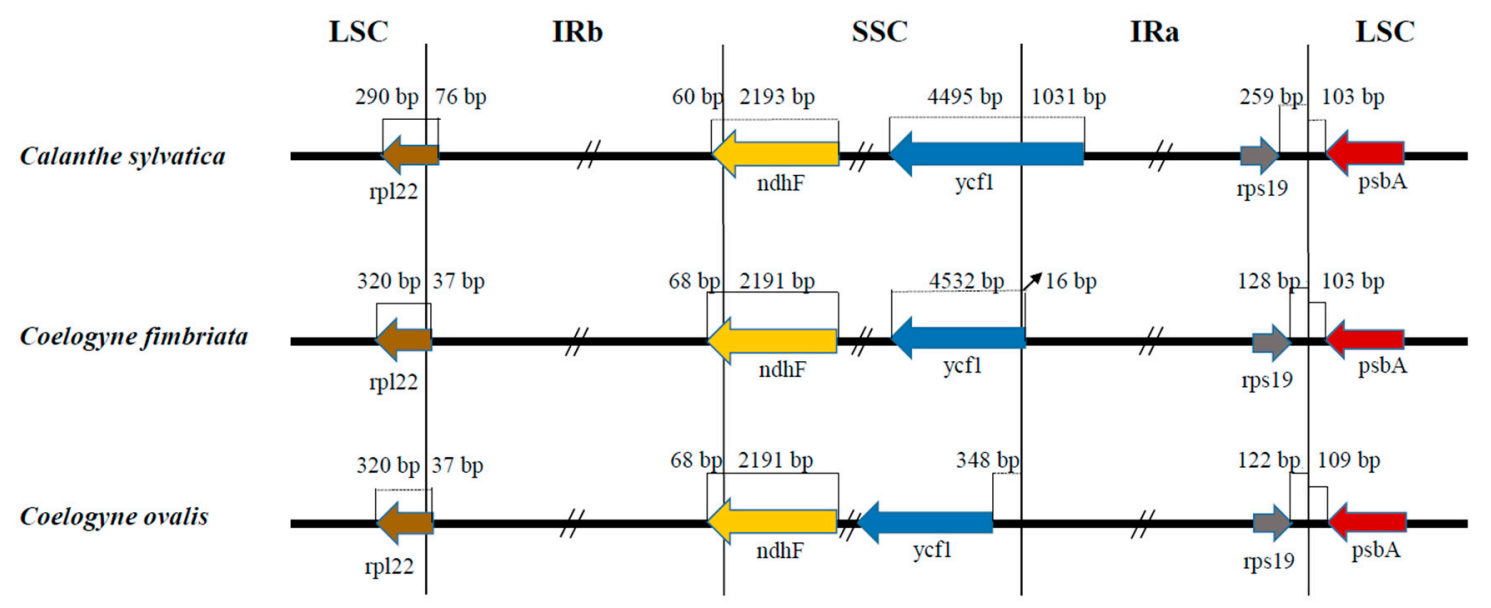

Figure 4. Borders comparison of the LSC, SSC, and IRs regions of two Coelogyne species with C. sylvatica as a reference. LSC: large single-copy region; SSC: small single-copy region; IRa: inverted repeat region $\mathrm{A} ; \mathrm{IRb}$ : inverted repeat region $\mathrm{B}$.

\subsection{Phylogenetic Position of Coelogyne in Orchidaceae}

To gain a clear insight into the phylogenetic position of C. fimbriata and C. ovalis, we carried out a phylogenetic analysis, with an aligned data matrix of the complete cp genome sequences of 67 orchid species. After removing ambiguous sites, we used 44,582 nucleotides to construct a phylogenetic tree using the Maximum Likelihood and Bayesian methods. Both results of the two methods indicated the same systematic relationship within Orchidaceae (e.g., (Vanilloideae [Orchidoideae, Epidendroideae])). It also showed the close relationship among Pleione, Bletilla, and Coelogyne with high bootstrap support (100) and posterior probability (1.00), which belong to the subtribe Coelogyninae Benth (Figure 5). 


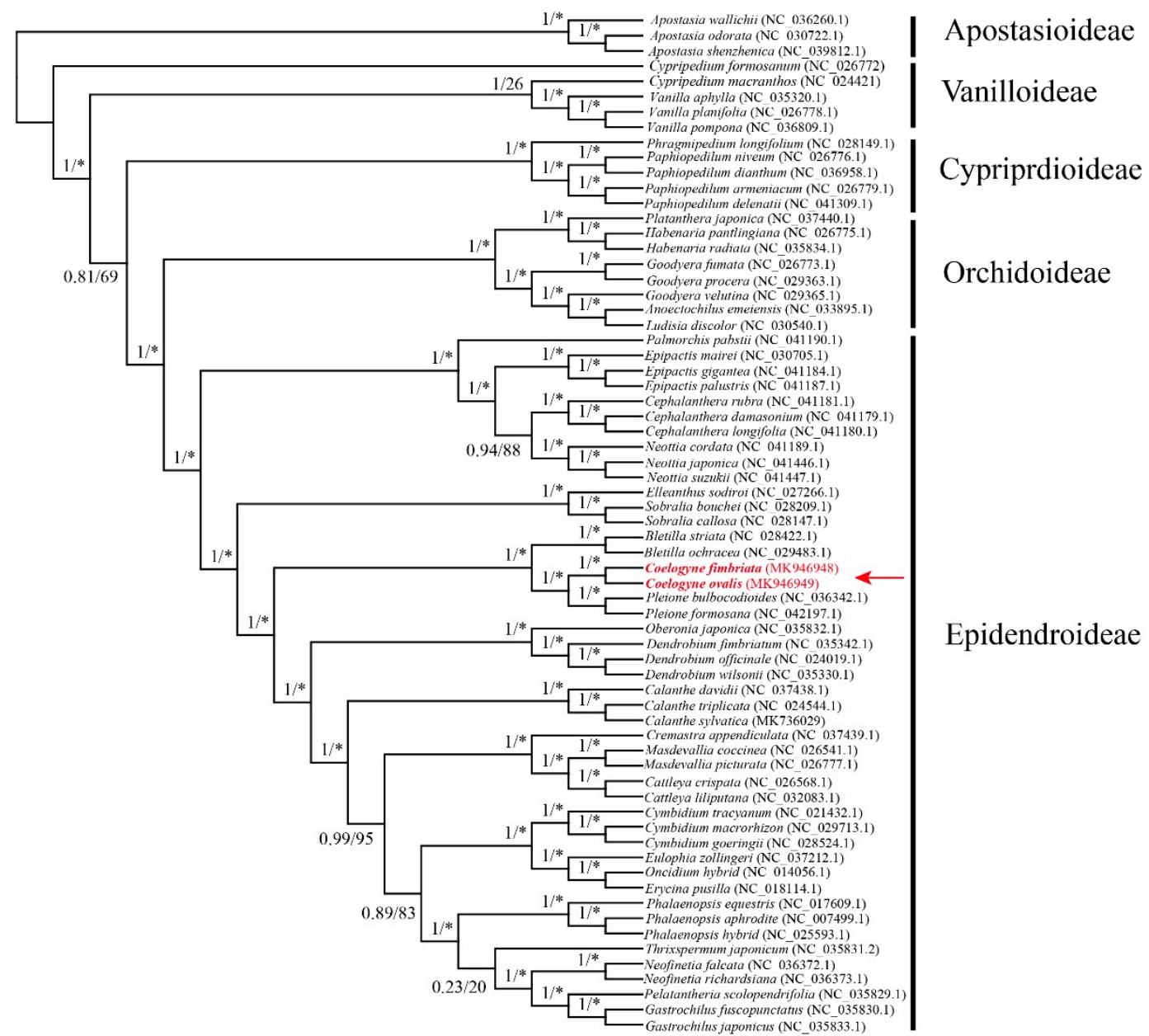

Figure 5. The phylogenic relationship of 67 Orchidaceae species with Maximum Likelihood (ML) and Bayesian analysis. * indicated 100 percent of bootstrap support using ML analysis.

\subsection{Primer Verification and Transferability}

We developed eight primers based on the medium variable regions within the LSC regions to compare the whole chloroplast genomes between $C$. fimbriata and $C$. ovalis. These primers were verified in 18 species of Coelogyne, including C. fimbriata and C. ovalis. Most Coelogyne species can be amplified using the eight primers (Table A1). All the sequences which were successfully amplified have been submitted to GenBank (Table A1).

\subsection{Phylogenetic Relationship within Coelogyne}

The alignments were $2858 \mathrm{bp}$ and $5719 \mathrm{bp}$ in the four- and eight-sequence matrix, respectively. When we considered the gap and missing data, a total of 128 and 302 polymorphic sites can be found, and the nucleotide diversity $\left(P_{\mathrm{i}}\right)$ was 0.0133 and 0.0099 in the four- and eight-sequence matrix among the 18 Coelogyne species. There were 42 parsimony informative sites within the above two alignments. According to Coelogyne's phylogenetic tree results based on four and eight fragments, two clades can be clustered with high bootstrap support (Figure 6). However, the interspecies relationship was conflicted between the two trees. Furthermore, we found that $C$. fimbriata and $C$. ovalis have the closest evolutionary relationship of all the species investigated (Figure 6). Using more Coelogyne species based only on matK sequence, a phylogenetic tree showed a low bootstrap support. In addition, the relationship between C. fimbriata and C. ovalis is still close (Figure A2). 

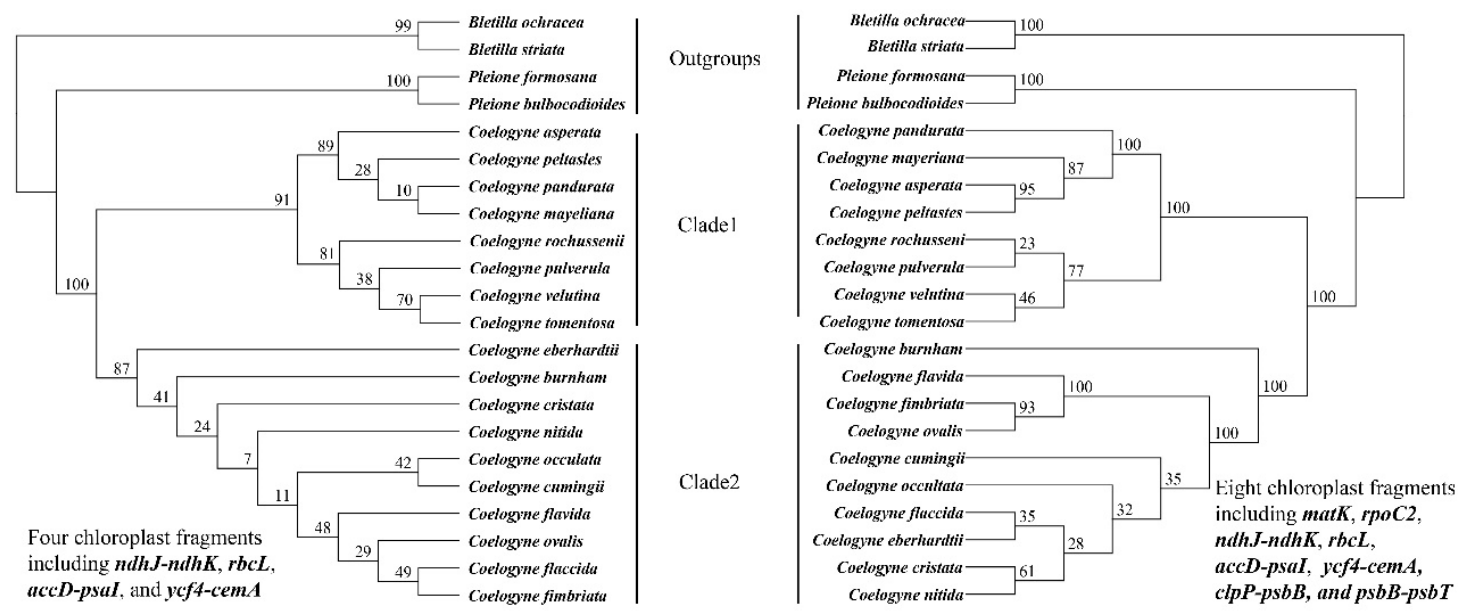

Figure 6. The Phylogenic relationship of 18 Coelogyne species with Maximum Likelihood (ML) analysis using four (left) and eight (right) chloroplast fragments, respectively. The number in the node showed the bootstrap support in the ML method using the RAxML software.

\section{Discussion}

\subsection{Coelogyne Chloroplast Genome Structure and Characterization}

In the angiosperms, most cp genomes are ordinarily conserved with a length of 120-160 kb and a content of 100-130 genes, but some Orchidaceae species' chloroplast genomes lost genes and rearrange structures [13]. In the current study, the cp genomes of $C$. fimbriata and $C$. ovalis each had 132 genes, consisting of 86 protein-coding genes, 38 tRNA genes, and eight rRNA genes. Moreover, the cp genome lengths of C. fimbriata and C. ovalis were $159,795 \mathrm{bp}$ and 160,040 bp, respectively. This length was consistent with most angiosperms, including the Orchidaceae. There are 74 protein-coding genes shared by all angiosperms, while several other genes, such as ycf1, ycf2, ycf4, rpl22, rpl23, rps16, ndhF, $a c c D$, and $\inf A$, are present in only some other species [14-18], with variation also observed in the Orchidaceae. We found that genes with a high frequency of absence from orchid species were usually $n d h K, n d h F, n d h E, n d h I, n d h A, y c f 15, y c f 1$, and $p s b G$, whereas genes with a low frequency of absence from orchid species were $n d h G, n d h D$, and infA [19-22]. Compared with other Epidendroideae species, the $p s b G$ gene was absent from the C. fimbriata and C. ovalis cp genomes [23]. The previous study showed that the $n d h$ genes were present in the common ancestor of orchids but have experienced independent, significant losses at least eight times in Orchidaceae [24]. This loss may be correlated in part with the unusual life history of orchids [24]. In this study, it is unknown whether the $p s b G$ gene was successfully transferred to the nucleus or completely lost from the entire cell of these two species, nor was this known for the other lost genes listed above. Combined with the reason of loss in other Epidendroideae species, we speculate that this may be related to the long-term evolution of genes to adapt to extreme living environments and climatic conditions, such as high altitude for C. ovalis and C. fimbriata, which could provide us with useful information concerning the dynamics of genetic evolution.

Repeat sequences could be used to study genome recombination and rearrangement [25]. In the present study, 43 repeat sequences were detected in the cp genome of both Coelogyne spp. Of the four types of repetition possible, most of those in C. fimbriata and C. ovalis were palindromic (P) repeats and forward (F) repeats, with percentages of $58 \%$ and $42 \%$, respectively, in C. fimbriata, and $60 \%$ and $30 \%$, respectively, in C. ovalis. Repeat sequence analysis of some other orchid species takes into account only these two repeat types ( $\mathrm{P}$ and $\mathrm{F}$ ) regardless of the other ones ( $\mathrm{C}$ and $\mathrm{R}$ ) [26]. This type suggests that palindromic and forward repeats are not only typical but representative in plants. Most repeat motifs existed in the IGS regions that play an essential role in the dynamic historical analysis of plant 
populations [27]. Furthermore, these data will provide us with specific insights into the phylogeny and evolutionary process of these Coelogyne species.

SSRs are widely distributed in eukaryotic genomes, consisting of tandem repeated sequences of 1-6 nucleotide motifs as the basic repeat unit. We identified 50 SSR loci in C. fimbriata, among which $86 \%$ were in the non-coding regions, with 35 in the IGS and eight in intron regions. In C. ovalis, on the other hand, a total of 48 SSR loci were detected, among which $90 \%$ were present in the non-coding regions, with 32 in IGS and 11 in intron regions. These results indicated that most of the polymorphisms were within the IGS regions, a finding which was consistent with earlier studies showing that the cp genome repeats were often present in non-coding regions, especially in IGS regions [28,29]. These data will provide us with tremendous help in further studying genetic diversity and population structure in the Orchidaceae.

The contraction and expansion of the SSC and IR boundary regions have been regarded as mechanisms by which the length difference within the angiosperm cp genome was achieved [30]. In the current study, a comparison of IR boundaries in two Coelogyne species was carried out, using C. sylvatica, which we had sequenced before, as a reference genome (Figure A1). The results showed that those genes close to the boundary line experienced shifts to different extents, which were mainly caused by the expansion of the four regions, which, in turn, were associated with differences in genome length comparisons among these three cp genomes (Figure 4). Moreover, the length of these genes has also changed. For example, the gene of $r p l 22$ and ycf1 had shortened, whereas the length of the $n d h F$ gene had increased (Figure A1). According to others, this expansion and contraction usually tended to be slight and even caused the duplication of parts of or even entire genes, which usually produced pseudogenes at the boundary of IR/SSC [30]. However, this situation did not occur in the cp genomes of C. fimbriata and C. ovalis. The related data are still preliminary, and it will be necessary to obtain more information to elucidate the mechanism by which variation in gene length occurred.

\subsection{Phylogenetic Analysis of Inter- and Intra- Coelogyne}

With the rise of the high-throughput sequencing and accurate assembly technology, chloroplast genomes are inexpensive and easy to obtain [31]. Phylogenomic studies using chloroplast genomes shed light on a more innovative and profound view than single or multiple genes in the systematic evolution [30]. To construct the phylogeny tree and determine Coelogyne's systematic position, we ultimately chose 67, from 28 genera, out of 122 species in the Orchidaceae, for which the full cp genome sequencing had been accomplished and officially published in the database of the NCBI. The results showed that the main relationship was the same as other studies among Vanilloideae, Orchidoideae, and Epidendroideae [32]. Within Epidendroideae, the relationship among tribes was ultimately the same as other studies using chloroplast genome CDS (coding sequence) [32]. These results showed that a systematic evolutionary relationship was robust using chloroplast genomes. Our focus genus Coelogyne and the Pleione form a high support clade (1.00 and 100 for BS and ML analysis) (Figure 5). The above clade and Bletilla clustered into a monophyletic tribe Arethuseae. The three genera's systematic relationship was in line with the previous study using the restriction fragment length polymorphism (RFLP), matK, and ITS markers, but our phylogenomic tree showed higher support [12]. Based on the above analysis, we inferred the close relationship between the Coelogyne and Pleione.

Within Coelogyne, we used the eight chloroplast fragments to construct a phylogenetic tree, including 18 Coelogyne species and four outgroup species. The eight newly developed primers showed high transferability, identifying high levels of variation among Coelogyne (Figure A1). The results revealed two high-support clades within Coelogyne (Clade1 and Clade2 in Figure 6, 100 bootstrap support for ML analysis), consistent with previous studies [12]. However, the relationship among the species within each clade was different from the earlier studies [12]. On one side, there are only four shared species between ours and the previous research. It was hard to compare the different phylogenetic trees with distinct species. On the other side, most clades have high support in our 
analysis using the ML method. In the future, more Coelogyne species can be added into the phylogenetic tree using the eight chloroplast fragments, which will provide a global view of the evolutionary relationship of Coelogyne.

Combining NCBI data and our new sequencing $m a t K$, we constructed a phylogenetic tree, including 82 Coelogyne species. However, bootstrap support is very low in most nodes (Figure A2). The results indicated the low resolution if only one chloroplast fragment is used. More chloroplast fragments are needed to construct a robust phylogenetic tree. Chloroplast genome resources provide a potential molecular marker for the study of systematic evolution.

\section{Materials and Methods}

\subsection{Plant Sampling and DNA Extraction}

We collected fresh leaves of Coelogyne fimbriata and Coelogyne ovalis from Jiangxi and Yunnan Provinces in China, respectively (Table A2). Approximately $50 \mathrm{~g}$ of fresh leaves of each species were sterilized with $75 \%$ ethanol and clean with distilled water, and then these materials were stored in a $4{ }^{\circ} \mathrm{C}$ refrigerator prior to further processing. The total chloroplast genomic DNA was extracted according to the high-salt methods provided by Shi et al. 2012 [33]. Approximately $1 \mu \mathrm{g}$ of DNA was prepared and processed to construct a DNA library according to the Illumina Sample Preparation Instructions using UltraTM DNA Library Prep Kit (New England Biolabs Inc., Ipswich, MA, USA). The cpDNA sample from each species was subjected to single-read sequencing with insertion lengths of $500 \mathrm{bp}$, using the Illumina MiSeq system (Illumina, San Diego, CA, USA). In addition, we collected leaf material of another 16 Coelogyne species from Shanghai Chenshan Botanical Garden (Table A2). Total DNA were extracted from the leaves using the Plant Genomic DNA Kit (TIANGEN Co., Ltd., Beijing, China).

\subsection{Genome Assembly and Annotation}

For each of the two species, low-quality reads were discarded from the raw reads, using Trimmomatic v0.39 [34] and Kmernator v1.0 software [35]. We mapped the clean reads to the reference cp genome of Calanthe sylvatica (GenBank accession no. MK736029) [36] with Burrows-Wheeler Aligner (BWA) v0.6 software [37]. The consensus sequences were extracted, and gaps were filled by polymerase chain reaction (PCR), with the primers designed based on the conserved sequences. According to the reference $\mathrm{cp}$ genome, the four LSC/IRs and SSC/IRs junctions of each of the two Coelogyne individuals were validated by PCR-based Sanger sequencing, using four pairs of primers. We used Dual Organellar GenoMe Annotator (DOGMA) software to initially annotate the chloroplast genomes [38]. These annotations were manually corrected for a start and stop codons and intron/exon boundaries by comparison with homologous genes in the Calanthe sylvatica cp genome. The tRNA genes were also verified by tRNAscan-SE v2.0 [39]. MAFFT v7.45 software [40] was employed to align the two Coelogyne cp genomes by comparing the structure and gene content. The online OGDRAW v1.3.1 program [41] was used to draw the two Coelogyne species' circular cp genomes.

\subsection{Repeat Sequence Analysis}

Perl script MISA v2.1 [42] was used to detect microsatellites, including mono-, di-, tri-, tetra-, penta-, and hexa-nucleotide repeats. We set the thresholds at ten repeat units for mononucleotide microsatellites or simple sequence repeats (SSRs) and five repeat units for di-, tri-, tetra-, penta-, and hexa-nucleotide SSRs. The REPuter software [43] was employed to visualize forward, palindrome, reverse, and complementary sequences. The criteria of a minimum repeat size were set as $30 \mathrm{bp}$, and the sequence identity was set as higher than $90 \%$. 


\subsection{Comparative Genome Analysis}

To identify divergence hotspots within Coelogyne cp genomes, we conducted a sliding window analysis to evaluate the nucleotide diversity $\left(P_{\mathrm{i}}\right)$ over the genomes, using DnaSP v5.10 software [44]. The window length and the step size were set to 600 and $200 \mathrm{bp}$, respectively. Genome, protein-coding gene, intron, and spacer sequence divergences were evaluated using DnaSP v5.10 [44], after alignment using MAFFT v7.45 software [40]. The chloroplast genome comparison between the two species was performed with the mVISTA program [45].

\subsection{Phylogenetic Position of the Two Coelogyne Species}

To determine the two Coelogyne species' systematic position, we performed a phylogenetic analysis using the whole $\mathrm{cp}$ genomes. In addition to the two Coelogyne $\mathrm{cp}$ genomes, we obtained another $65 \mathrm{cp}$ genome sequences, representing different lineages of Orchidaceae from the National Center for Biotechnology Information (NCBI) Organelle Genome Resource database. Three species in the genus Apostasia were set as the outgroups among these 67 taxa. First, we used MAFFT v.7.45 software [40] to align the 67 chloroplast genomes, setting the gap open penalty and offset value as 1.53 and 0.12 , respectively. Second, Gblocks v0.91b software [46] was used to refine the alignment with allowed gap positions set as none. This software can eliminate poorly aligned positions and divergent regions. After selecting the best-fitting model of nucleotide substitution for the entire dataset (GTRGAMA) (Table A3), as determined by the Akaike Information Criterion (AIC) in MEGA X [47], the Maximum Likelihood (ML) and Bayesian (BI) analyses were performed in RAxML-HPC v8.2.11 software [48] and MrBayes v3.2 software [49], respectively. The ML analysis searches for the best trees, starting from 1000 random trees, and bootstrap percentages were obtained with 1000 non-parametric bootstrap replicates. In the BI analysis, we run the Markov chain Monte Carlo (MCMC) algorithm with two independent chains using a random starting tree and default priors for 1,000,000 generations, with trees sampled every 1000 generations. We assumed the convergence of the MCMC chains after the average standard deviation of split frequencies reached 0.01 or less. We performed ML and BI analysis on the Cyberinfrastructure for Phylogenetic Research (CIPRES) Science Gateway website v3.3 (http://www.phylo.org/).

\subsection{Primers Design and Verification in Other Coelogyne Species}

To develop more effective primers for medicinal plant identification and phylogeny analysis, we designed eight pairs of primers (Figure 7, Table 4), based on the conserved sequences on both sides of the medium variable regions within the large single-copy (LSC) regions. These primers were used to amplify and carry out Sanger sequencing of the two species and another 16 Coelogyne species (Table A2). First, we used these sequences to validate the two cp genomes' accuracy by comparing eight fragments and genome sequences. Second, the efficiency of the newly developed markers was tested using these 18 Coelogyne species.
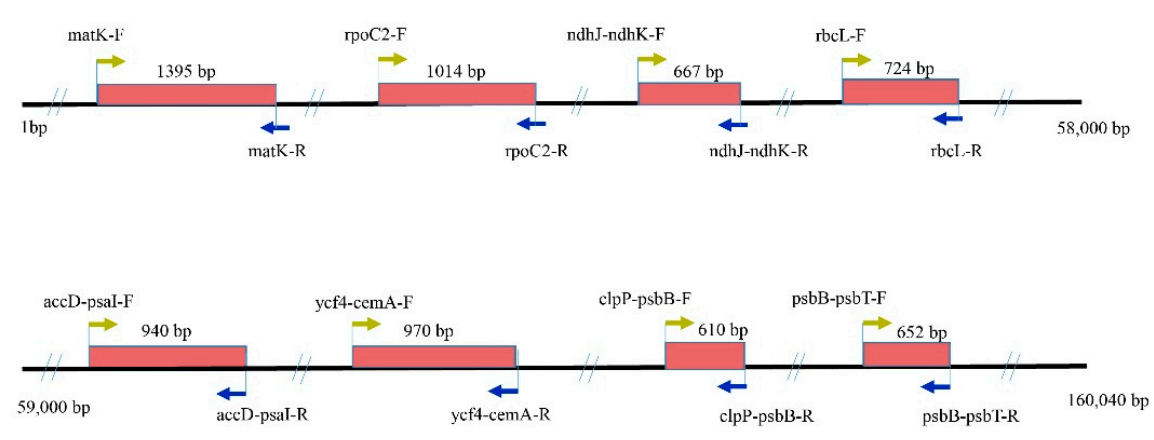

Figure 7. The relative positions of eight designed primers in two Coelogyne species. The arrow indicates the location and direction of the primer, which is amplified specifically. The rectangles in the red indicate the length of those products. 
Table 4. Basic Information of Eight Chloroplast Primers.

\begin{tabular}{|c|c|c|c|c|}
\hline Locus & Primer Sequence $\left(5^{\prime}-3^{\prime}\right)$ & Location & $\begin{array}{c}\text { Product } \\
\text { Length (bp) }\end{array}$ & $\begin{array}{c}\text { Annealing } \\
\text { Temperature/Tm }\left({ }^{\circ} \mathrm{C}\right)\end{array}$ \\
\hline \multirow{2}{*}{ matK } & F: CACCAGATCATTGATACGGA & CDS & 1395 & 55 \\
\hline & R: CCTGTGGAAATTCTCGGTTA & & & \\
\hline \multirow{2}{*}{ rpoC2 } & F: TATTGTCCATGCCTCTTCAC & CDS & 1014 & 55 \\
\hline & R: CATTTTTCTGGAGAGGTGGA & & & \\
\hline \multirow{2}{*}{$n d h J-n d h K$} & F: CCTATCCAACTTTCAGGCAT & IGS & 667 & 55 \\
\hline & R: ATCACAAGTTTGACCTTCGA & & & \\
\hline \multirow{2}{*}{$r b c L$} & F: TCGAGTAGACCTTGTTGTTG & IGS & 724 & 55 \\
\hline & R: CGGCACAAAATAAGAAACGA & & & \\
\hline \multirow{2}{*}{$a c c D-p s a I$} & F: TGTTTTCTTTGGGGACATCA & IGS & 940 & 55 \\
\hline & R: CGGAAAGGCCACATATCATA & & & \\
\hline \multirow{2}{*}{$y c f 4-c e m A$} & F: TGAGAATTTGACTCCACGAG & IGS & 970 & 55 \\
\hline & R: ATTTCGGATTGCCTGGTATT & & & \\
\hline \multirow{2}{*}{$c l p P-p s b B$} & F: ACACCAATGGGCATTAAGAT & IGS & 610 & 55 \\
\hline & R: ACCTGTTCGGTAGATTTTGT & & & \\
\hline \multirow{2}{*}{$p s b B-p s b N$} & F: ATGCTCAAGTGGAATTTGGA & IGS & 652 & 55 \\
\hline & R: GAACTTTAGGTGGTTCTCGA & & & \\
\hline
\end{tabular}

CDS: coding region; IGS: intergenic spacer.

\subsection{Phylogenetic Relationship within Coelogyne}

To determine the 18 species' divergence hotspot, we used DNAsp v.5.10 [44] software to calculate the number of variable sites and nucleotide diversity among the 18 species. Because some Coelogyne species failed to obtain all eight fragments, we created two sequence matrices. One sequence matrix includes four fragments ( $n d h J-n d h K, r b c L$, accD-psaI, and ycf4-cem $A$ ) shared by all 18 species, and another sequence matrix consisting of eight fragments with some missing data (Table A1). We constructed a phylogenetic tree using two sequence matrixes. We selected Bletiall striata, Bletiall ochracea, Pleione formosana, Pleione bulbocodioides as the outgroup species. We extracted the same sequence fragments of the eight primers' locations after alignment with MAFFT v7.45 [40] from the whole chloroplast genome of the above four species; then, the four or eight fragments of four species were combined like all other sequences using SequenceMatrix v1.7.8 [50]. Gblocks v0.91b [46] was used to refine the alignment with allowed gap positions set as none. Phylogenetic analysis of two sequence matrices was conducted by RAxML-HPC v8.2.11 [48] using the generalised time reversible with shape parameter of the gamma distribution (GTRGAMA) model. We searched for the best trees by starting from 1000 random trees, and bootstrap percentages were obtained with 1000 non-parametric bootstrap replicates.

We also downloaded 239 matK sequences from the NCBI database. After removing too short and duplicate-species sequences, we obtained a total of 89 sequences (including 14 sequences from this study) and aligned these sequences representing 82 Coelogyne species. We chose P. formosana and P. bulbocodioides as the outgroup. After alignment using MAFFT v7.45 [40], Gblocks v0.91b [46] was used to refine the alignment with allowed gap positions set as none. Using the same parameters as the above analysis, we constructed a phylogenetic tree using RAxML-HPC v8.2.11 [48] using the GTRGAMA model. We searched for the best trees by starting from 1000 random trees, and bootstrap percentages were obtained with 1000 non-parametric bootstrap replicates.

\section{Conclusions}

To our knowledge, this was the first study to characterize the chloroplast genome of the potentially medicinal plants $C$. fimbriata and $C$. ovalis. The new cpDNA sequences will provide useful information for developing molecular markers. The results increase Coelogyne's genomic data and provide fundamental references for further studies of the Coelogyneae tribe. Such genetic information can 
provide additional knowledge to support the conservation or the horticultural or phytopharmaceutical exploitation of these two Himalayan orchids.

Author Contributions: All authors contributed to the study conception and design. K.J., L.-Y.M., and Z.-W.W. contributed to the material preparation. K.J., Z.-Y.N., X.-H.Z., and C.H. contributed to the data collection. The analysis were performed by K.J., C.H., and W.-C.H. The first draft of the manuscript was written by K.J., L.-Y.M., and W.-C.H., and all authors commented on previous versions of the manuscript. All authors have read and agreed to the published version of the manuscript.

Funding: This study was funded by the project of Shanghai Landscaping and City Appearance Administrative Bureau to Kai Jiang (grant number G182418) and Chao Hu (grant numbers G192424 and G202401). This study was also supported by grants from the Science and Technology Commission, Shanghai Municipality, to Wei-Chang Huang (grant number 19390743600).

Conflicts of Interest: The authors declare that they have no conflict of interest.

\section{Appendix A}

Table A1. GenBank Accession of Eight Chloroplast Fragments for 18 Coelogyne Species in the Study.

\begin{tabular}{|c|c|c|c|c|c|c|c|c|}
\hline \multirow{2}{*}{ Species } & \multicolumn{8}{|c|}{ GenBank Accession } \\
\hline & matK & rpoC2 & $n d h J-n d h K$ & $r b c L$ & $a c c D-p s a I$ & $y c f 4-\operatorname{cem} A$ & $c l p P-p s b B$ & $p s b B-p s b T$ \\
\hline C. rochussenii & - & - & MN512535 & MN416673 & MN512468 & MN512517 & MN512484 & - \\
\hline C. burnham & MN400405 & MN400397 & MN512520 & MN396950 & MN512453 & MN512502 & MN512471 & MN512487 \\
\hline C. veluting & MN416681 & MN416666 & MN512537 & MN416675 & MN512470 & MN512519 & MN512486 & MN512501 \\
\hline C. mayeliana & MN400412 & MN400404 & MN512528 & MN400420 & MN512461 & MN512510 & - & - \\
\hline C. peltasles & MN416679 & & MN512532 & MN416670 & MN512465 & MN512514 & MN512482 & MN512498 \\
\hline C. cumingii & MN400407 & MN400399 & MN512522 & MN400414 & MN512455 & MN512504 & MN512473 & MN512489 \\
\hline C. flavida & MN400409 & MN400401 & MN512525 & MN400417 & MN512458 & MN512507 & MN512476 & MN512492 \\
\hline C.eberhardtii & MN400408 & MN400400 & MN512524 & MN400416 & MN512457 & MN512506 & MN512475 & MN512491 \\
\hline C. cristata & - & - & MN512523 & MN400415 & MN512456 & MN512505 & MN512474 & MN512490 \\
\hline C. tomentosa & - & MN416665 & MN512536 & MN416674 & MN512469 & MN512518 & MN512485 & MN512500 \\
\hline C. occulata & MN416678 & MN416661 & MN512531 & MN416669 & MN512464 & MN512513 & MN512481 & MN512497 \\
\hline C. flaccida & MN400411 & MN400403 & MN512527 & MN400419 & MN512460 & MN512509 & MN512478 & MN512494 \\
\hline C. pulverula & MN416680 & MN416663 & MN512534 & MN416672 & MN512467 & MN512516 & MN512483 & MN512499 \\
\hline C. asperata & MN400406 & MN400398 & MN512521 & MN400413 & MN512454 & MN512503 & MN512472 & MN512488 \\
\hline C. pandurata & - & MN416662 & MN512533 & MN416671 & MN512466 & MN512515 & - & - \\
\hline C. nitida & MN416676 & MN416659 & MN512529 & MN416667 & MN512462 & MN512511 & MN512479 & MN512495 \\
\hline C. fimbriata & MN400410 & MN400402 & MN512526 & MN400418 & MN512459 & MN512508 & MN512477 & MN512493 \\
\hline C. ovalis & MN416677 & MN416660 & MN512530 & MN416668 & MN512463 & MN512512 & MN512480 & MN512496 \\
\hline
\end{tabular}

- indicate the failed PCR.

Table A2. Specimen Information for the Coelogyne Spp. Samples Used in This Study.

\begin{tabular}{ccccc}
\hline Species & Collector & Collection No. & Deposited Institution & $\mathbf{n}$ \\
\hline C. fimbriata & Wei-Chang Huang & CS-HWC201606-2 & CSH & 1 \\
C. ovalis & Wei-Chang Huang & CS-HWC201606-5 & CSH & 1 \\
C. rochussenii & Kai Jiang & CS-JK201806-01 & CSH & 1 \\
C. burnham & Kai Jiang & CS-JK201806-02 & CSH & 1 \\
C. veluting & Kai Jiang & CS-JK201806-03 & CSH & 1 \\
C. mayeliana & Kai Jiang & CS-JK201806-04 & CSH & 1 \\
C. peltasles & Kai Jiang & CS-JK201806-05 & CSH & 1 \\
C. cumingii & Kai Jiang & CS-JK201806-06 & CSH & 1 \\
C. flavida & Kai Jiang & CS-JK201806-07 & CSH & 1 \\
C. eberhardtii & Kai Jiang & CS-JK201806-08 & CSH & 1 \\
C. cristata & Kai Jiang & CS-JK201806-09 & CSH & 1 \\
C. tomentosa & Kai Jiang & CS-JK201806-10 & CSH & 1 \\
C. occulata & Kai Jiang & CS-JK201806-11 & CSH & 1 \\
C. flaccida & Kai Jiang & CS-JK201806-12 & CSH & 1 \\
C. pulverula & Kai Jiang & CS-JK201806-13 & CSH & 1 \\
C. asperata & Kai Jiang & CS-JK201806-14 & CSH & 1 \\
C. pandurata & Kai Jiang & CS-JK201806-15 & CSH & 1 \\
C. nitida & Kai Jiang & CS-JK201806-16 & CSH & 1 \\
\hline
\end{tabular}

All voucher specimens were deposited in shanghai chenshan herbarium (CSH), shanghai, China. all the materials were collected in living plants from Shanghai Chenshan Botanical Garden. $\mathrm{n}$ showed the number of collected sample. 
Table A3. Best model selection based on the Maximum Likelihood method.

\begin{tabular}{cccccccc}
\hline Model & Param & BIC & AICc & lnL & Invariant & Gamma & R \\
\hline GTR + G & 140.00 & $582,962.94$ & $581,155.75$ & $-290,437.87$ & n/a & 0.94 & 1.39 \\
GTR + G + I & 141.00 & $582,977.87$ & $581,157.77$ & $-290,437.88$ & 0.00 & 0.94 & 1.39 \\
T92 + G & 134.00 & $585,265.34$ & $583,535.60$ & $-291,633.79$ & n/a & 0.93 & 1.48 \\
TN93 + G & 137.00 & $585,287.36$ & $583,518.89$ & $-291,622.44$ & n/a & 0.93 & 1.48 \\
HKY + G & 136.00 & $585,287.51$ & $583,531.95$ & $-291,629.97$ & n/a & 0.93 & 1.48 \\
T92 + G + I & 135.00 & $585,403.95$ & $583,661.30$ & $-291,695.64$ & 0.00 & 0.93 & 1.57 \\
TN93 + G + I & 138.00 & $585,425.88$ & $583,644.51$ & $-291,684.25$ & 0.00 & 0.92 & 1.57 \\
HKY + G + I & 137.00 & $585,426.37$ & $583,657.91$ & $-291,691.95$ & 0.00 & 0.93 & 1.57 \\
GTR + I & 140.00 & $587,343.22$ & $585,536.03$ & $-292,628.01$ & 0.31 & n/a & 1.37 \\
T92 + I & 134.00 & $589,665.50$ & $587,935.76$ & $-293,833.87$ & 0.31 & n/a & 1.34 \\
HKY + I & 136.00 & $589,688.10$ & $587,932.55$ & $-293,830.27$ & 0.31 & n/a & 1.34 \\
TN93 + I & 137.00 & $589,690.94$ & $587,922.48$ & $-293,824.23$ & 0.31 & n/a & 1.34 \\
K2 + G & 133.00 & $591,575.20$ & $589,858.37$ & $-294,796.18$ & n/a & 0.84 & 1.56 \\
K2 + G + I & 134.00 & $591,756.46$ & $590,026.72$ & $-294,879.35$ & 0.00 & 0.83 & 1.65 \\
GTR & 139.00 & $592,361.70$ & $590,567.41$ & $-295,144.70$ & n/a & n/a & 1.33 \\
T92 & 133.00 & $594,707.63$ & $592,990.80$ & $-296,362.39$ & n/a & n/a & 1.32 \\
HKY & 135.00 & $594,729.93$ & $592,987.28$ & $-296,358.63$ & n/a & n/a & 1.32 \\
TN93 & 136.00 & $594,733.26$ & $592,977.71$ & $-296,352.85$ & n/a & n/a & 1.32 \\
K2 + I & 133.00 & $596,345.39$ & $594,628.56$ & $-297,181.27$ & 0.33 & n/a & 1.45 \\
JC + G & 132.00 & $600,630.85$ & $598,926.93$ & $-299,331.46$ & n/a & 0.87 & 0.50 \\
JC + G + I & 133.00 & $600,645.77$ & $598,928.94$ & $-299,331.46$ & 0.00 & 0.87 & 0.50 \\
K2 & 132.00 & $602,173.44$ & $600,469.52$ & $-300,102.75$ & n/a & n/a & 1.39 \\
JC + I & 132.00 & $605,419.13$ & $603,715.21$ & $-301,725.60$ & 0.32 & n/a & 0.50 \\
JC & 131.00 & $610,996.30$ & $609,305.29$ & $-304,521.64$ & n/a & n/a & 0.50 \\
\hline
\end{tabular}

Models with the lowest Bayesian Information Criterion (BIC scores) are considered to describe the substitution pattern the best. For each model, the Akaike Information Criterion, corrected (AICc) value, Maximum Likelihood value (lnL), and the number of parameters (including branch lengths) are also presented. Non-uniformity of evolutionary rates among sites may be modeled by using a discrete Gamma distribution $(+G)$ with five rate categories and by assuming that a certain fraction of sites is evolutionarily invariable $(+\mathrm{I})$. Whenever applicable, estimates of the gamma shape parameter and the estimated fraction of invariant sites are shown. Assumed or estimated values of transition/transversion bias (R) are shown for each model, as well. For estimating ML values, a tree topology was automatically computed. This analysis involved 67 nucleotide sequences. There were a total of 44,582 positions in the final dataset. Evolutionary analyses were conducted in MEGA X. 
Calanthe sylvatica Coelogyne fimbria
Criteria: $70 \%, 100$ bp

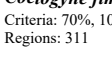

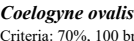

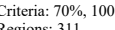

$$
\text { (a) }
$$
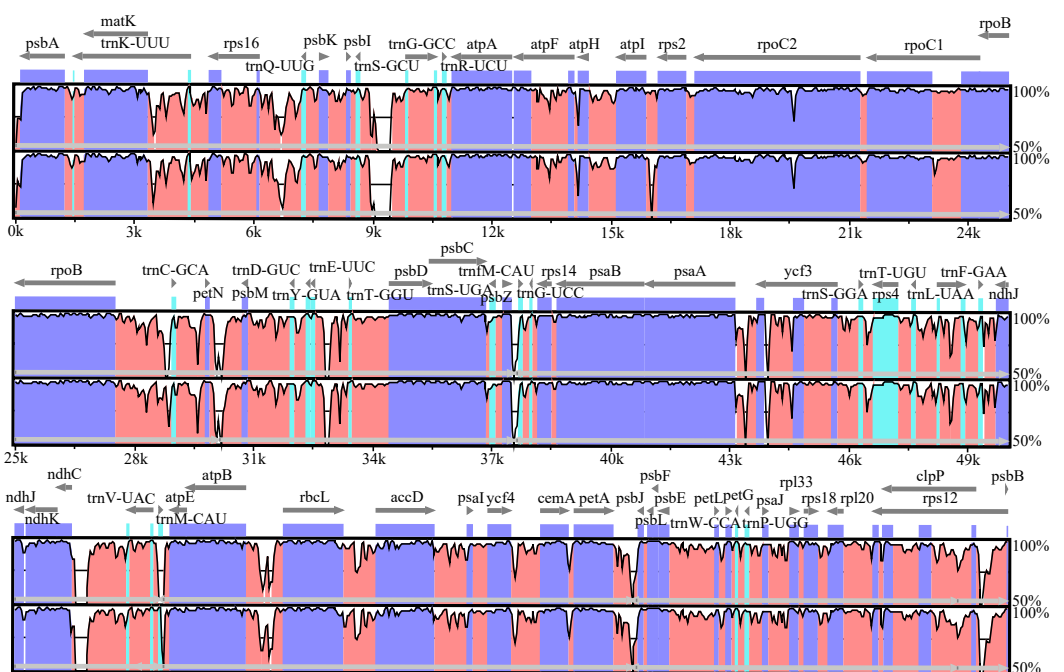

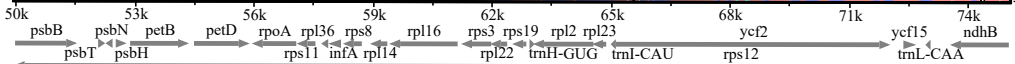
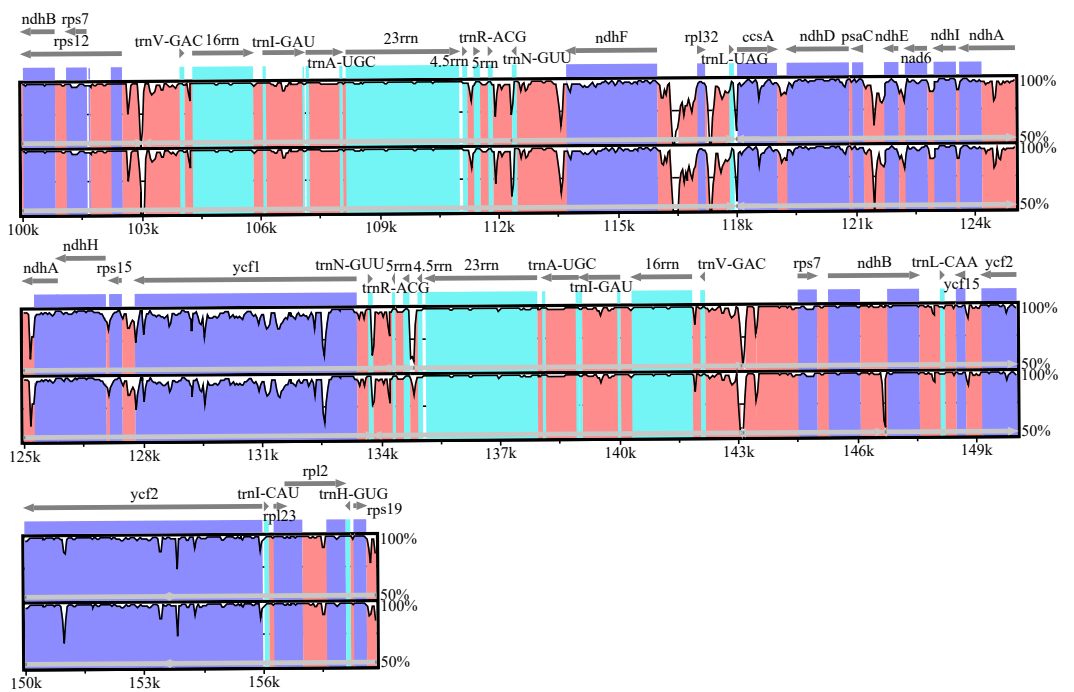

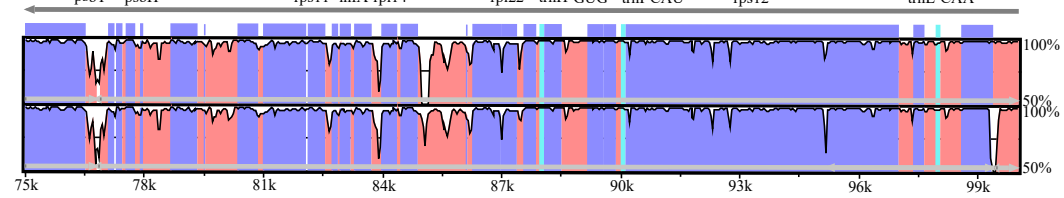

Figure A1. The cp genome sequence comparison of two Coelogyne species with Calanthe sylvatica as a reference. Dark grey arrows show the direction and position of genes. Pink and dark blue areas show Conserved Non-coding Sequences (CNS) and exon regions, respectively. The untranslated regions (UTRs) are colored with light-blue, including tRNA and rRNA regions. The peaks and valleys show the percent of conservation with an identity cutoff of $50 \%$. 


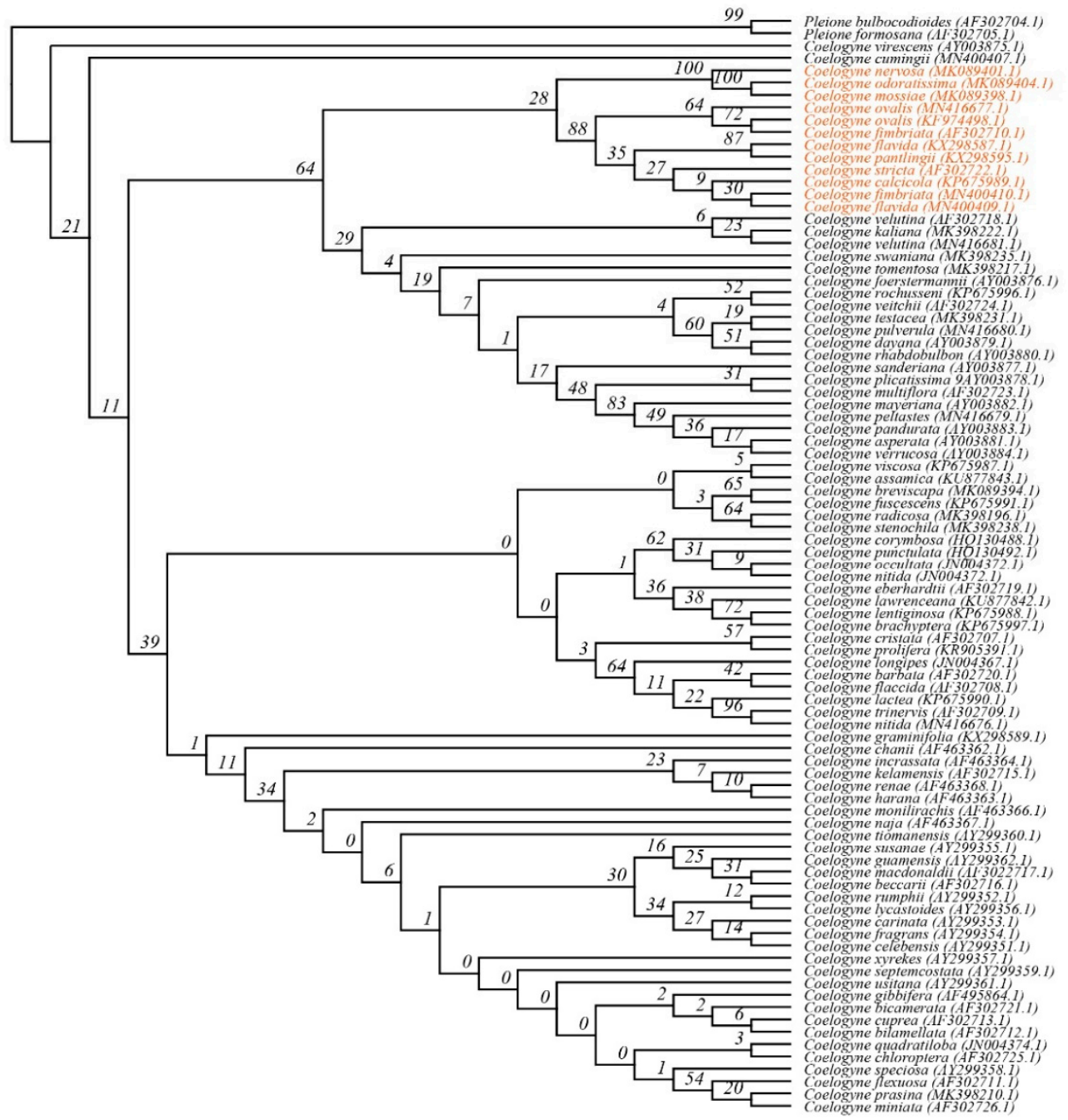

Figure A2. Phylogenetic tree based on 89 matK sequences representing 82 Coelogyne species using two Pleione species as outgroup. The number in the node showed the bootstrap support in the ML method using RAxML.

\section{References}

1. Dyall, S.D.; Brown, M.T.; Johnson, P.J. Ancient invasions: From endosymbionts to organelles. Science 2004, 304, 253-257. [CrossRef] [PubMed]

2. Chumley, T.W.; Palmer, J.D.; Mower, J.P.; Fourcade, H.M.; Calie, P.J.; Boore, J.L.; Jansen, R.K. The complete chloroplast genome sequence of Pelargonium $\times$ hortorum: Organization and evolution of the largest and most highly rearranged chloroplast genome of land plants. Mol. Biol. Evol. 2006, 23, 2175-2190. [CrossRef] [PubMed]

3. Palmer, J.D.; Jansen, R.K.; Michaels, H.J.; Chase, M.W.; Manhart, J.R. Chloroplast DNA variation and plant phylogeny. Ann. Mo. Bot. Gard. 1988, 1180-1206. [CrossRef]

4. Corriveau, J.L.; Goff, L.J.; Coleman, A.W. Plastid DNA is not detectable in the male gametes and pollen tubes of an angiosperm (Antirrhinum majus) that is maternal for plastid inheritance. Curr. Genet. 1990, 17, 439-444. [CrossRef]

5. Chen, X.Q.; Clayton, D. Coelogyne Lindley. In Flora of China; Wu, Z.Y., Raven, P.H., Eds.; 25; Science Press, Beijing \& Missouri Botanical Garden Press: St. Louis, MO, USA, 2009; pp. 315-325.

6. Cheng, J.; Shi, J.; Shangguan, F.Z.; Dafni, A.; Deng, Z.H.; Luo, Y.B. The pollination of a self-incompatible, food-mimic orchid, Coelogyne fimbriata (Orchidaceae), by female Vespula wasps. Ann. Bot. 2009, 104, 565-571. [CrossRef] 
7. Satake, M.; Ijung, L. Flowers in Myanmar (Part II): Wild orchid and medicinal orchid. Aroma Res. 2004, 5, 83-89.

8. Pramanick, D.D. Pharmacognostic studies on the pseudobulb of Coelogyne cristata Lindl.(Orchidaceae)-An epiphytic orchid of ethno-medicinal importance. J. Pharmacogn. Phytochem. 2016, 5, 120.

9. Singh, N.; Kumaria, S. A Combinational Phytomolecular-Mediated Assessment in Micropropagated Plantlets of Coelogyne ovalis Lindl.: A Horticultural and Medicinal Orchid. Proc. Natl. Acad. Sci. India Sect. B Biol. Sci. 2020, 90, 455-466. [CrossRef]

10. Teoh, E.S. Medicinal orchids of Asia; Springer: Cham, Switzerland, 2016.

11. Wu, X.R. A Concise Edition of Medicinal Plants in China; Guangdong Higher Education Publication House: Guangdong, China, 1994. (In Chinese)

12. Gravendeel, B.; Chase, M.W.; de Vogel, E.F.; Roos, M.C.; Mes, T.H.; Bachmann, K. Molecular phylogeny of Coelogyne (Epidendroideae; Orchidaceae) based on plastid RFLPs, matK, and nuclear ribosomal ITS sequences: Evidence for polyphyly. Am. J. Bot. 2001, 88, 1915-1927. [CrossRef] [PubMed]

13. Lin, C.S.; Chen, J.J.; Chiu, C.C.; Hsiao, H.C.; Yang, C.J.; Jin, X.H.; Leebens-Mack, J.; de Pamphilis, C.W.; Huang, Y.T.; Yang, L.H.; et al. Concomitant loss of NDH complex-related genes within chloroplast and nuclear genomes in some orchids. Plant J. 2017, 90, 994-1006. [CrossRef]

14. Hiratsuka, J.; Shimada, H.; Whittier, R.; Ishibashi, T.; Sakamoto, M.; Mori, M.; Kondo, C.; Honji, Y.; Sun, C.R.; Meng, B.Y. The complete sequence of the rice (Oryza sativa) chloroplast genome: Intermolecular recombination between distinct tRNA genes accounts for a major plastid DNA inversion during the evolution of the cereals. Mol. Gen. Genet. MGG 1989, 217, 185-194. [CrossRef]

15. Maier, R.M.; Neckermann, K.; Igloi, G.L.; Kössel, H. Complete sequence of the maize chloroplast genome: Gene content, hotspots of divergence and fine tuning of genetic information by transcript editing. J. Mol. Biol. 1995, 251, 614-628. [CrossRef]

16. Gantt, J.S.; Baldauf, S.L.; Calie, P.J.; Weeden, N.F.; Palmer, J.D. Transfer of $r p l 22$ to the nucleus greatly preceded its loss from the chloroplast and involved the gain of an intron. EMBO J. 1991, 10, 3073-3078. [CrossRef] [PubMed]

17. Thomas, F.; Massenet, O.; Dome, A.M.; Briat, J.F.; Mache, R. Expression of the $r p l 23$, rpl2 and rps19 genes in spinach chloroplasts. Nucleic Acids Res. 1988, 16, 2461-2472. [CrossRef] [PubMed]

18. Nagano, Y.; Matsuno, R.; Sasaki, Y. Sequence and transcriptional analysis of the gene cluster trnQ-zfpA-psaI-ORF231-petA in pea chloroplasts. Curr. Genet. 1991, 20, 431-436. [CrossRef] [PubMed]

19. Wu, F.H.; Chan, M.T.; Liao, D.C.; Hsu, C.T.; Lee, Y.W.; Daniell, H.; Duvall, M.R.; Lin, C.S. Complete chloroplast genome of Oncidium Gower Ramsey and evaluation of molecular markers for identification and breeding in Oncidiinae. BMC Plant Biol. 2010, 10, 68. [CrossRef] [PubMed]

20. Roma, L.; Cozzolino, S.; Schlüter, P.M.; Scopece, G.; Cafasso, D. The complete plastid genomes of Ophrys iricolor and O. sphegodes (Orchidaceae) and comparative analyses with other orchids. PLOS ONE 2018, 13, e0204174. [CrossRef]

21. Pan, I.C.; Liao, D.C.; Wu, F.H.; Daniell, H.; Singh, N.D.; Chang, C.; Shih, M.C.; Chan, M.T.; Lin, C.S. Complete chloroplast genome sequence of an orchid model plant candidate: Erycina pusilla apply in tropical Oncidium breeding. PLoS ONE 2012, 7, e34738. [CrossRef]

22. Yang, J.B.; Tang, M.; Li, H.T.; Zhang, Z.R.; Li, D.Z. Complete chloroplast genome of the genus Cymbidium: Lights into the species identification, phylogenetic implications and population genetic analyses. BMC Evol. Biol. 2013, 13, 84. [CrossRef]

23. Li, J.; Chen, C.; Wang, Z.Z. The complete chloroplast genome of the Dendrobium strongylanthum (Orchidaceae: Epidendroideae). Mitochondrial DNA Part A 2016, 27, 3048-3049. [CrossRef] [PubMed]

24. Kim, H.T.; Kim, J.S.; Moore, M.J.; Neubig, K.M.; Williams, N.H.; Whitten, W.M.; Kim, J.H. Seven new complete plastome sequences reveal rampant independent loss of the $n d h$ gene family across orchids and associated instability of the inverted repeat/small single-copy region boundaries. PLoS ONE 2015, 10, e0142215. [CrossRef] [PubMed]

25. Cavalier-Smith, T. Chloroplast evolution: Secondary symbiogenesis and multiple losses. Curr. Biol. 2002, 12, R62-R64. [CrossRef] 
26. Yao, X.; Tang, P.; Li, Z.; Li, D.; Liu, Y.; Huang, H. The first complete chloroplast genome sequences in Actinidiaceae: Genome structure and comparative analysis. PLOS ONE 2015, 10, e0129347. [CrossRef] [PubMed]

27. Small, R.L.; Ryburn, J.A.; Cronn, R.C.; Seelanan, T.; Wendel, J.F. The tortoise and the hare: Choosing between noncoding plastome and nuclear $A d h$ sequences for phylogeny reconstruction in a recently diverged plant group. Am. J. Bot. 1998, 85, 1301-1315. [CrossRef] [PubMed]

28. Provan, J.; Powell, W.; Hollingsworth, P.M. Chloroplast microsatellites: New tools for studies in plant ecology and evolution. Trends Ecol. Evol. 2001, 16, 142-147. [CrossRef]

29. Jakobsson, M.; Säll, T.; Lind-Halldén, C.; Halldén, C. Evolution of chloroplast mononucleotide microsatellites in Arabidopsis thaliana. Theor. Appl. Genet. 2007, 114, 223. [CrossRef]

30. Kim, K.J.; Lee, H.L. Complete chloroplast genome sequences from Korean ginseng (Panax schinseng Nees) and comparative analysis of sequence evolution among 17 vascular plants. DNA Res. 2004, 11, 247-261. [CrossRef]

31. Vu, H.T.; Tran, N.; Nguyen, T.-D.; Vu, Q.L.; Bui, M.H.; Le, M.T.; Le, L. Complete chloroplast genome of Paphiopedilum delenatii and phylogenetic relationships among Orchidaceae. Plants 2020, 9, 61. [CrossRef]

32. Kim, Y.K.; Jo, S.; Cheon, S.H.; Joo, M.J.; Hong, J.R.; Kwak, M.; Kim, K.J. Plastome evolution and phylogeny of Orchidaceae, with 24 new sequences. Front. Plant Sci. 2020, 11, 22. [CrossRef]

33. Shi, C.; Hu, N.; Huang, H.; Gao, J.; Zhao, Y.J.; Gao, L.Z. An improved chloroplast DNA extraction procedure for whole plastid genome sequencing. PLoS ONE 2012, 7, e31468. [CrossRef]

34. Bolger, A.M.; Lohse, M.; Usadel, B. Trimmomatic: A flexible trimmer for Illumina sequence data. Bioinformatics 2014, 30, 2114-2120. [CrossRef] [PubMed]

35. Egan, R. Kmernator: An MPI Toolkit for Large Scale Genomic Analysis. 2014. Available online: https: //github.com/JGI-Bioinformatics/Kmernator (accessed on 8 October 2020).

36. Miao, L.Y.; Hu, C.; Huang, W.C.; Jiang, K. Chloroplast genome structure and phylogenetic position of Calanthe sylvatica (Thou.) Lindl. (Orchidaceae). Mitochondrial DNA Part B 2019, 4, 2625-2626. [CrossRef]

37. Li, H.; Durbin, R. Fast and accurate long-read alignment with Burrows-Wheeler transform. Bioinformatics 2010, 26, 589-595. [CrossRef]

38. Wyman, S.K.; Jansen, R.K.; Boore, J.L. Automatic annotation of organellar genomes with DOGMA. Bioinformatics 2004, 20, 3252-3255. [CrossRef]

39. Lowe, T.M.; Eddy, S.R. tRNAscan-SE: A program for improved detection of transfer RNA genes in genomic sequence. Nucleic Acids Res. 1997, 25, 955-964. [CrossRef]

40. Katoh, K.; Standley, D.M. MAFFT multiple sequence alignment software version 7: Improvements in performance and usability. Mol. Biol. Evol. 2013, 30, 772-780. [CrossRef]

41. Stephan, G.; Pascal, L.; Ralph, B. OrganellarGenomeDRAW (OGDRAW) version 1.3.1: Expanded toolkit for the graphical visualization of organellar genomes. Nucleic Acids Res. 2019, 47, 59-64. [CrossRef]

42. Thiel, T.; Michalek, W.; Varshney, R.; Graner, A. Exploiting EST databases for the development and characterization of gene-derived SSR-markers in barley (Hordeum vulgare L.). Theor. Appl. Genet. 2003, 106, 411-422. [CrossRef]

43. Kurtz, S.; Choudhuri, J.V.; Ohlebusch, E.; Schleiermacher, C.; Stoye, J.; Giegerich, R. REPuter: The manifold applications of repeat analysis on a genomic scale. Nucleic Acids Res. 2001, 29, 4633-4642. [CrossRef] [PubMed]

44. Librado, P.; Rozas, J. DnaSP v5: A software for comprehensive analysis of DNA polymorphism data. Bioinformatics 2009, 25, 1451-1452. [CrossRef] [PubMed]

45. Frazer, K.A.; Pachter, L.; Poliakov, A.; Rubin, E.M.; Dubchak, I. VISTA: Computational tools for comparative genomics. Nucleic Acids Res. 2004, 32, W273-W279. [CrossRef]

46. Talavera, G.; Castresana, J. Improvement of phylogenies after removing divergent and ambiguously aligned blocks from protein sequence alignments. Syst. Biol. 2007, 56, 564-577. [CrossRef] [PubMed]

47. Kumar, S.; Stecher, G.; Li, M.; Knyaz, C.; Tamura, K. MEGA X: Molecular Evolutionary Genetics Analysis across computing platforms. Mol. Biol. Evol. 2018, 35, 1547-1549. [CrossRef] [PubMed]

48. Stamatakis, A. RAxML version 8: A tool for phylogenetic analysis and post-analysis of large phylogenies. Bioinformatics 2014, 30, 1312-1313. [CrossRef] [PubMed] 
49. Huelsenbeck, J.P.; Ronquist, F. MRBAYES: Bayesian inference of phylogenetic trees. Bioinformatics 2001, 17, 754-755. [CrossRef]

50. Vaidya, G.; Lohman, D.J.; Meier, R. SequenceMatrix: Concatenation software for the fast assembly of multi-gene datasets with character set and codon information. Cladistics 2011, 27, 171-180. [CrossRef]

(C) 2020 by the authors. Licensee MDPI, Basel, Switzerland. This article is an open access article distributed under the terms and conditions of the Creative Commons Attribution (CC BY) license (http://creativecommons.org/licenses/by/4.0/). 